\title{
Cloud camera design using a Raspberry Pi
}

\section{Diseño de una cámara de nubes usando Raspberry Pi}

\author{
J. C. Antuña-Sánchez1, N. Díaz1, R. Estevan1, A. M. de Frutos2S, J. C. Antuña-Marrero1 \\ 1. Atmospheric Optics Group of Camagüey (GOAC), Camagüey Meteorological Center, Camagüey, Cuba. \\ 2. Atmospheric Optics Group (GOA), Valladolid University, Valladolid, Spain \\ (*) E-mail: jcantuna@goac.cu S: S: miembro de SEDOPTICA / SEDOPTICA member \\ Received / Recibido: 19/05/2015 Accepted / Aceptado: 26/08/2015 \\ DOI: 10.7149/OPA.48.3.199
}

\begin{abstract}
:
The design and assembly of low cost all-sky camera for clouds detection is presented. The instrument comply with all the requirements currently established for this type of instrument. Under the conditions of Cuba, it is impossible to acquire such a device, which costs between $\$ 600$ and $\$ 3500$ USD. Using a Raspberry Pi, its camera module with a CMOS sensor and a unipolar stepper motor (recovered from a discontinued matrix printer) we have built a sky camera for less than \$300 USD. The Raspberry Pi, using free software and hardware, will control and conduct the operation of the camera, the image capturing, the processing and the transmission of the latter results. Among the advantages provided for this device stand objectively determining the percentages of sky covered by clouds, the ability to archive images taken for potential future reprocessing, the classifications of clouds according to the attenuation of solar radiation they produce, among others. All this advantages will be achieved with an instrument of very low cost, allowing access to this technology for both research networks and meteorological services in poor countries.
\end{abstract}

Key words: all-sky camera, raspberry pi, cloud camera

\section{RESUMEN:}

Se presenta el diseño y montaje de una cámara de cielo para la detección de nubes, de bajo costo, pero con las prestaciones necesarias según los más actuales requerimientos para este tipo de instrumento. En las condiciones de Cuba resulta imposible adquirir un dispositivo de este tipo cuyo precio oscila entre los €600 y €3500 EUR. Usando una Raspberry Pi, el módulo de cámara fotográfica con sensor CMOS y un motor paso a paso unipolar (recuperado de una impresora matricial descontinuada) hemos construido una cámara de cielo con un costo inferior a los €300 EUR. La operación de la cámara, la captura de imágenes, su procesamiento y el envío de los resultados de este último, lo realizará el mismo equipo utilizando software y hardware libre. Entre las ventajas previstas para este dispositivo se destacan la determinación en forma objetiva de los porcientos de cielo cubierto por nubes, la posibilidad de archivar las imágenes tomadas para ser sometidas a futuro reprocesamiento, potenciales clasificaciones de las nubes según la atenuación de la radiación solar que producen, entre otras. Todo ello será obtenido con un equipo de muy bajo costo lo que permitiría a los servicios meteorológicos de países subdesarrollados o con escasos recursos, el acceso a esta tecnología.

Palabras clave: cámara de cielo, raspberry pi, cámara de nubes

\section{REFERENCES AND LINKS / REFERENCIAS Y ENLACES}

[1] OMM, Reglamento Técnico. Volumen 1: Normas meteorológicas de carácter general y prácticas recomendadas. Edición 2011, Actualizada 2012. Reporte OMM-49. ISBN 92-63-39049-5, 55 pp (2012).

[2] WMO, Guide to meteorological instruments and methods of observations. WMO No.8, $567 \mathrm{pp}$ (2006).

[3] Fassig, O., A revolving cloud camera. Mon. Wea. Rev., 43 (6), 274-275 pp (1915). 
[4] Anthes, R., A. Robock, J. C. Antuña-Marrero, O. García, J. J. Braun, and R. Estevan Arredondo, 2015: Cooperation on GPS Meteorology between the United States and Cuba. Bull. Amer. Meteor. Soc., 96, 1079-1088 (2015) http://dx.doi.org/10.1175/BAMS-D-14-00171.1

[5] Estevan,R., L. Mona, N. Papagiannopoulos, J. C. Antuña, V. Cachorro and A. de Frutos, 2014, CALIPSO and sunphotometer measurements of Saharan Dust events over Camagüey. Opt. Pura Apl. 47 (3), pp. 189-196 (2014) http://dx.doi.org/10.7149/OPA.47.3.189

[6] Antuña, J. C., R. Estevan, B. Barja, 2012: Demonstrating the Potential for First-Class Research in Underdeveloped Countries: Research on Stratospheric Aerosols and Cirrus Clouds Optical Properties, and Radiative Effects in Cuba (1988-2010). Bull. Amer. Meteor. Soc., 93, 1017-1027. http://dx.doi.org/10.1175/BAMS-D-11-00149.1

[7] Raspberry, Raspberry Pi Foundation, http//:www.raspberrypi.org (2015).

[8] F.M. Wanlass and C.T. Sah: "Nanowatt Logic Using Field-Effect Metal. Oxide Semiconductor Triodes", Solid-State Circuits Conference. Digest of Technical Papers. 1963 IEEE International, 32 - 33 (1963). http://dx.doi.org/10.1109/isscc.1963.1157450

[9] Hain, R., C. J. Kähler, C. Tropea, 2007: Comparison of CCD, CMOS and intensified cameras, Experiments in Fluids, Vol. 42, Issue 3, pp 403-411. http://dx.doi.org/10.1007/s00348-006-0247-1

[10] Uhl, A., 2015: Image Processing and Computer Vision. Course notes WS 2013/2014, 136 pp. http://www.cosy.sbg.ac.at/ uhl/IPCV.pdf

[11] Hill, R., A lens for whole sky photographs. Quarterly Journal of the Royal Meteorological Society 50:227-235 pp (1924).

[12] A. Cazorla, F. J. Olmo, and L. Alados-Arboledas, "Development of a sky imager for cloud cover assessment," J. Opt. Soc. Am. A 25, 29-39 (2008). http://dx.doi.org/10.1364/JOSAA.25.000029

[13] I. Reda and A. Andreas, "Solar Position Algorithm for Solar Radiation Applications," National Renewable Energy Laboratory, NREL/TP-560-34302.

\section{Introducción}

La nubosidad desempeña un papel predominante en la regulación de la cantidad de radiación solar que alcanza la superficie terrestre. Existen diversos instrumentos que realizan observaciones de la distribución espacio-temporal de las nubes. En la actualidad entre los más comunes se destacan los radares meteorológicos, lidares y radiómetros tanto en la superficie como a bordo de satélites. No obstante, las observaciones realizadas desde las estaciones convencionales de superficie sobre la distribución espacial de las nubes siguen siendo hoy de gran utilidad tanto para el cumplimiento de las tareas asignadas a los servicios meteorológicos como para la investigación científica. En el primero de estos casos, la OMM (Organización Meteorológica Mundial) incluye la "nubosidad fraccional" (que es una forma de expresar la "cobertura nubosa total", término que se empleará de aquí en adelante) como uno de los datos necesarios para todos los programas de la OMM y los servicios meteorológicos nacionales [1].

Históricamente la estimación de la cobertura nubosa total, observada desde un punto en la superficie terrestre con fines de la representación sinóptica del estado de la atmósfera, se ha realizado de forma visual por el observador meteorológico con intervalos de 3 horas en condiciones de tiempo normales. El observador es entrenado para realizar la división de la bóveda celeste en 8 o 10 partes (octas o décimos respectivamente) para estimar la cantidad de ellos que están cubiertos por nubes [2]. Las observaciones resultantes tienen un fuerte componente subjetivo.

La primera referencia a una cámara de nubes encontrada en la literatura se remite a una cámara rotatoria que componía la imagen de $360^{\circ}$ de la bóveda celeste por sectores, construida en 1905 [3]. A partir de entonces estos instrumentos han evolucionado y han alcanzado cierta difusión, mayormente en el campo de la Astronomía y en la Meteorología. Las cámaras de nubes menos costosas que hemos encontrado en el mercado son de $€ 600$ y únicamente permiten visualizar la imagen tomada en un monitor. No dispone del software que permita al usuario adquirir y procesar las imágenes, puesto que están destinadas a soportar la observación astronómica. Aproximadamente al doble de precio están disponibles otras con un simple 
software. Aquellas con el software más avanzado son desarrolladas por grupos de investigación a partir de cámaras comerciales y su costo puede alcanzar alrededor de los $€ 3,500$.

El desarrollo del Grupo de Óptica Atmosférica de Camagüey (GOAC) ha sido posible gracias a una estrategia en la que se combinan la máxima explotación de las capacidades profesionales del personal del grupo con la cooperación internacional [4]. Esta es la respuesta a la seria limitación que impone el hecho de que el país no dispone de financiamiento para la adquisición de equipamiento científico. La aplicación de la mencionada estrategia, en particular a través de la cooperación internacional, ha permitido al GOAC la creación de un sitio experimental que consta de instrumentos de última generación para el estudio de las propiedades ópticas de los aerosoles [5] y del contenido integral de vapor de agua [6]. El diseño, construcción y puesta en operación de una cámara de nubes por personal del GOAC, con cooperación internacional, es un nuevo esfuerzo para fortalecer la capacidad observacional instalada. Al contar solo con limitado financiamiento internacional para los componentes más importantes de la cámara, la adquisición de los componentes más baratos (pero que cumplen con los más elementales requisitos del instrumento) es determinante.

\section{Materiales y métodos}

\section{2.a. Raspberry Pi}

El núcleo de la cámara de nubes que se describe en el presente trabajo es un ordenador de placa única de bajo coste producido por la firma Raspberry. El modelo Raspberry Pi, que es el que seleccionamos, se emplea para la captura y posterior procesamiento de las imágenes, sin la necesidad de usar una computadora convencional o laptop para estos fines. La multifuncionalidad de la Raspberry Pi en el manejo de periféricos nos aporta un conjunto de posibilidades a la hora de diseñar la cámara, específicamente a la hora de añadir sensores y el manejo de un motor paso a paso unipolar para mover una barra que bloqueara el sol.

La Raspberry Pi se comenzó a comercializar en agosto de 2011 con el objetivo de ser utilizada para incentivar el conocimiento en el uso de la electrónica y la programación en edades tempranas [7]. Desde esa fecha ha transitado por 4 modelos, aumentando cada vez más su capacidad de procesamiento y prestaciones. El modelo utilizado para nuestro equipo es el B+, que cuenta con un procesador ARM a 700 MHz, 512 MB de memoria SDRAM (Synchronous Dynamic Random-Access Memory, en español, Memoria de Acceso Sincrónico, Dinámico y Aleatorio), salidas de video compuesto y HDMI (High-Definition Multimedia Interface, en español, Interface Multimedia de Alta Definición) hasta 1080p. Su almacenamiento es mediante tarjetas MicroSD; además posee un bajo consumo de energía llegando solamente a los $3.5 \mathrm{~W}$. Además cuenta con 40 pines de GPIO (General Purpose Input Output, en español, Entrada Salida de Propósito General) y el sistema operativo más usado para su operación es el Raspbian, una variante de Debian diseñada especialmente para ella.

Uno de los motivos que nos llevó al uso de la Raspberry Pi fue la variedad de dispositivos desarrollados para ella, entre ellos el módulo de cámara. Para este módulo ha sido desarrollada la librería Picamera, basada en Python y que permite controlar todo el proceso de operación, captura y almacenamiento de las imágenes tomadas. El sensor que usa esta cámara es del tipo CMOS (Complementary Metal-OxideSemiconductor, en español, Semiconductor Complementario de Óxido Metálico). En el siguiente subepígrafe abordamos el tema de los sensores de las cámaras digitales.

\section{2.b. Sensores CMOS y CCD}

El sensor de imagen de una cámara digital capta la luz que compone la imagen y la convierte en una señal, esta puede terminar en formato analógico o digital. En la actualidad los sensores más utilizados son el CCD (Charge Coupled Device, en español, Dispositivo de Carga Acoplada) y el CMOS, aunque en su esencia funcionan de manera similar, cada tecnología tiene sus particularidades.

Los sensores CCD contienen diminutas células fotoeléctricas, la cuales se basan en el efecto fotoeléctrico, que registran la imagen y a partir de allí un conversor análogo-digital, que no se integra en el mismo chip del sensor, se encarga de realizar el procesamiento y almacenamiento de la imagen.

Los sensores CMOS al igual que el CCD se basan en el efecto fotoeléctrico, con la diferencia que se incorpora un amplificador de la señal eléctrica y el conversor análogo digital se encuentra integrado dentro del mismo chip [8]. 
Establecer una comparación entre ambos sensores no puede hacerse en detalle porque las especificaciones del sensor y su rendimiento, normalmente no son brindadas por los fabricantes [9]. No obstante una comparación de las propiedades respectivas de estos sensores, se muestra a continuación [10]:

TABLA 1. Comparación entre CCD y CMOS

\begin{tabular}{|l|c|c|}
\hline \hline \multicolumn{1}{|c|}{ Parámetros } & CCD & CMOS \\
\hline \hline Señal del Pixel & Carga & Voltaje \\
\hline Señal del Chip & Voltaje & Bits \\
\hline Factor de Forma & Alto & Moderado \\
\hline Complejidad del Sistema & Alta & Baja \\
\hline Complejidad del Sensor & Baja & Alta \\
\hline Rango Dinámico & Muy Alto & Alto \\
\hline Consumo de Energía & Alto & Bajo \\
\hline \hline
\end{tabular}

En resumen, ninguno de los dos es completamente superior al otro, la tecnología ha hecho que el CMOS, en teoría algo inferior al CCD, se acerque bastante en calidad al CCD volviéndose casi imperceptible al ojo humano. El módulo de cámara desarrollado para Raspberry Pi cuenta con un sensor CMOS, esta cámara tiene las siguientes especificaciones:

TABLA 2. Especificaciones de la cámara para Raspberry Pi

\begin{tabular}{|c|c|}
\hline \hline Tamaño de imagen & 2592x1944 píxeles \\
\hline Resolución de video & 1080p a 30 FPS, 720p a 60 FPS y 640x480 a 60 ó 90 FPS \\
\hline Dimensiones & 25mm x 20mm x 9mm \\
\hline Peso & 3 gramos \\
\hline Precio & \$25 USD a \$ 30 USD \\
\hline
\end{tabular}

En las cámaras de cielo comerciales el sensor más utilizado es el CCD, pero sus precios son bastante elevados, al necesitar una mayor complejidad en la electrónica de la cámara y nuestro objetivo es desarrollar un dispositivo de bajo coste que se adapte a nuestras condiciones.

Para lograr una imagen que abarque la mayor parte de la bóveda celeste se emplea un objetivo ojo de pez o fish eye, cuya distorsión (deliberada) se asemeja a una imagen reflejada en una esfera. Este tipo de objetivo fue desarrollado para su uso en la meteorología en el estudio de las nubes [11]; de ellos existen 2 variedades, una que capta toda la superficie del sensor formando imágenes rectangulares y otra que forman la imagen circular, siendo el primero el que utilizaremos en nuestro diseño aunque no sea el ideal pero es el que pudimos obtener.

\section{2.c. Sensores, motor paso a paso unipolar y librería PySolar}

Teniendo en cuenta que el equipo va a estar expuesto a las condiciones ambientales y que además en el trópico la temperatura y la humedad relativa son altas, se decidió agregar un sensor que registrara estas variables, con el objetivo de monitorearlas y el envío de alarmas con el fin de evitar roturas en el equipo. Además estos valores que formarán parte de la metadata en cada observación y serán almacenados.

Uno de los problemas presentados en el proceso de diseño fue la incidencia de los rayos del sol y la saturación de la imagen producida por estos. En la literatura se reportan soluciones para el mismo, que es también el que se presenta en los instrumentos diseñados para medir radiación solar difusa. Por ejemplo, en la cámara de cielo instalada en el CEAMA (Centro Andaluz de Medio Ambiente) se utiliza un seguidor de sol de Kipp\&Zone, el cual se mueve en ambos ejes para ubicar una esfera en la posición exacta del sol, pero este sistema, dada su complejidad y exactitud, queda fuera de nuestras posibilidades [12]. Se decidió utilizar una barra que, dada la ubicación del sol, obstruyera la radiación solar directa a la hora de tomar la imagen y así garantizar una baja saturación.

Para poder ubicar la barra de obstrucción de la radiación solar directa, solo moviéndola en azimut y con exactitud en la posición requerida, se empleó un motor paso a paso unipolar recuperado de una impresora matricial descontinuada. Este motor tiene una amplitud en cada paso de $1.8^{\circ}$ pero una de las ventajas de estos motores es la posibilidad de moverlos medio paso con lo cual se logra una amplitud de $0.9^{\circ}$ por paso logrando una mayor exactitud. Todo esto se realiza mediante un controlador desarrollado para este fin, 
utilizando un arreglo de transistores Darlington ULN2003 y opto-acopladores para la protección de la Raspberry Pi.

Para el cálculo de la posición del sol se utiliza la librería PySolar, desarrollada en Python [13] y con buenos resultados en comparación con el algoritmo usado por el USNO (United States Naval Observatory).

\section{2.d. Régimen de trabajo}

Como abordamos anteriormente, las observaciones de nubosidad la realizan los observadores cada una hora, nos planteamos que el régimen de trabajo a realizar fuera una observación cada 5 minutos desde la salida hasta la puesta del sol. Todo este proceso se realizará de manera automática gracias a un software desarrollado en Python para este fin.

\section{Resultados}

Aunque el dispositivo se encuentra en la fase de puesta a punto se han obtenido algunos resultados, uno de ellos es la construcción de un controlador para manipular de forma segura el motor paso a paso, ya que la Raspberry Pi no cuenta con protecciones eléctricas. En las siguientes figuras se observa el montaje completo del sistema y su ubicación en el sitio instrumental del GOAC.

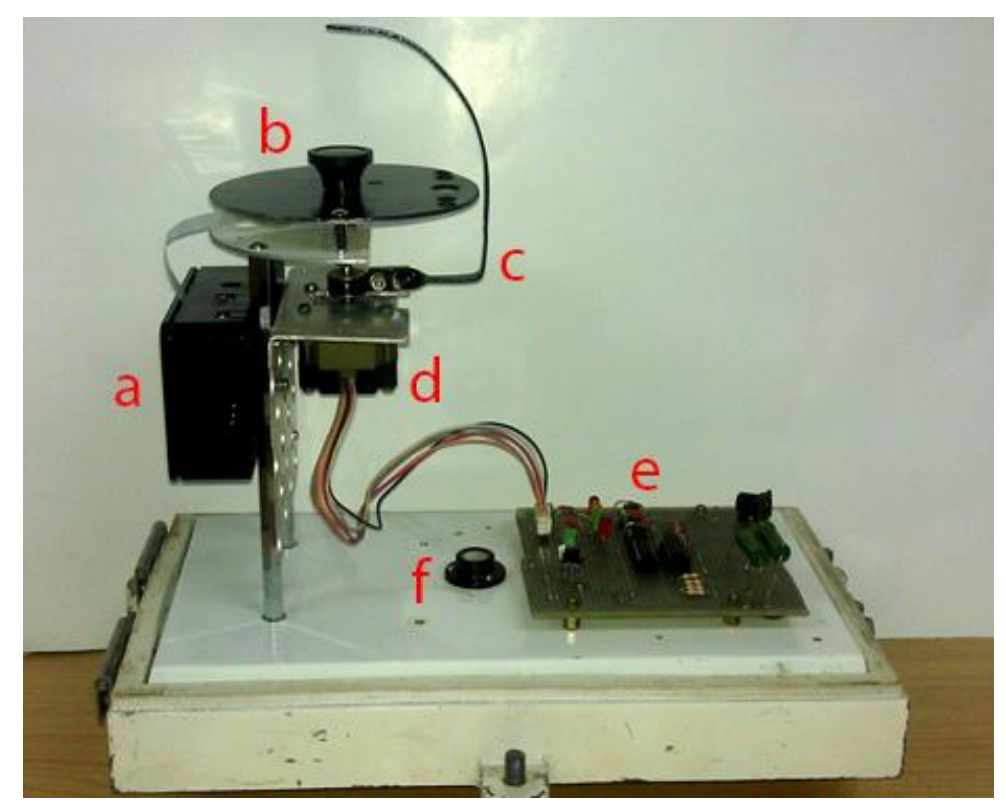

Fig.1. Montaje completo del sistema: a) Raspberry Pi; b) Cámara y lente "ojo de pez"; c) barra de obstrucción de la radiación solar directa; d) motor paso a paso; e) controlador del motor paso a paso; f) base de soporte de todo el sistema con nivel de burbuja.

Como se puede apreciar en la imagen la mayor parte del cielo está despejada y para ese día y hora el observador actinométrico reportó a las 9:12 (hora local) 4/10 de cielo cubierto, de ellos 3/10 nubes bajas. $\mathrm{Al}$ terminar nuestro prototipo procederemos a desarrollar el algoritmo para el cálculo del total de cielo cubierto, así como la clasificación de nubes en finas o gruesas.

Actualmente se trabaja en el desarrollo e implementación del software de control de la operación de la cámara de nubes y del procesamiento de sus observaciones. Una vez completada la primera versión, completamente operativa, de la cámara de nubes, se prevé incorporar sensores de temperatura y humedad así como, dispositivos para garantizar la estabilidad de ambas variables en el instrumento. Se explorará además la posibilidad de emplear los valores RGB de los pixeles para realizar la clasificación de los tipos de nubes.

Como resultado de nuestras primeras pruebas con la cámara y el objetivo de ojo de pez obtuvimos varias imágenes como la mostrada en la figura 2. 


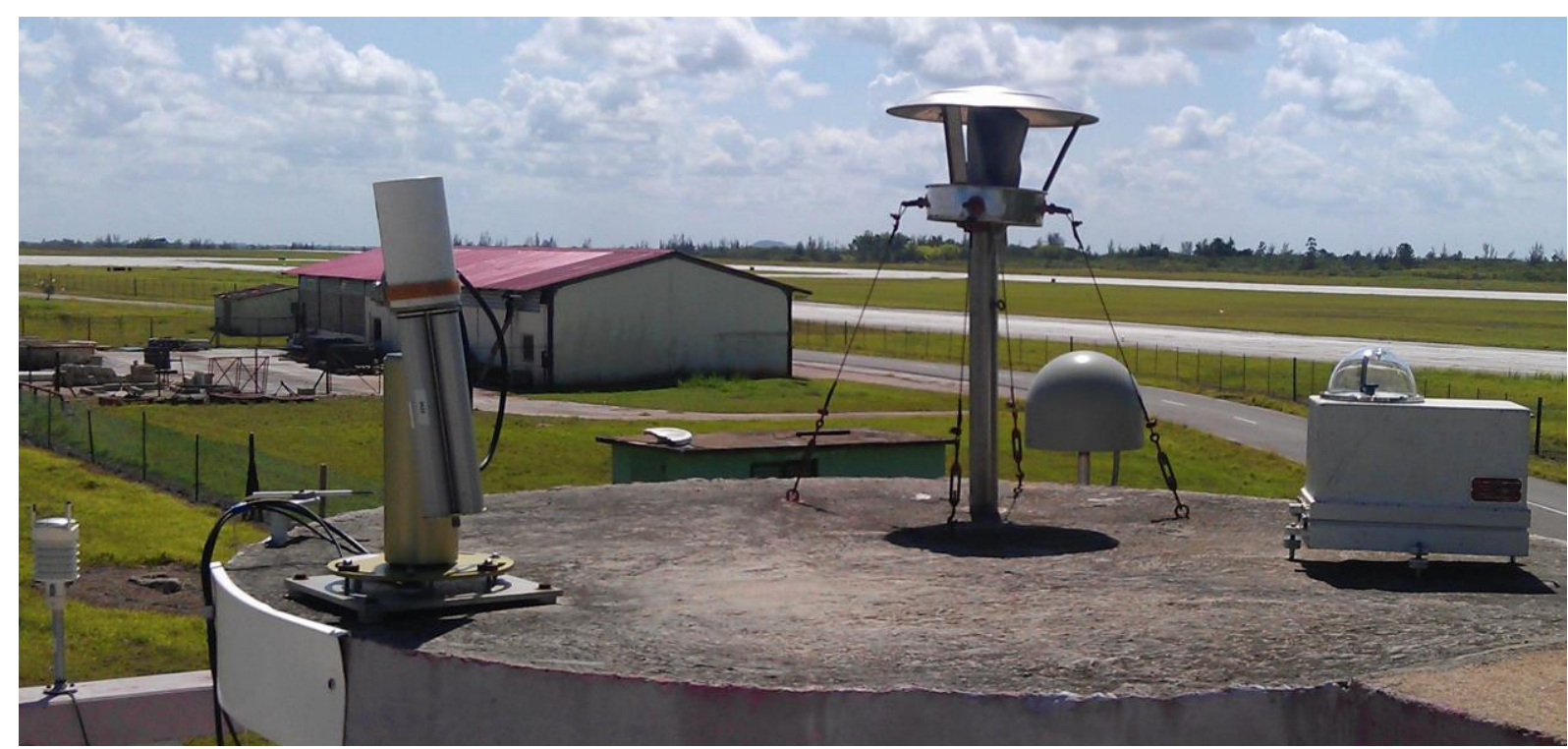

Fig.2. Ubicación del prototipo en la plazoleta instrumental del GOAC. Instrumentos (de izquierda a derecha): Estación Automática Vaisala WTX 520, Fotómetro Solar Cimel CE-318, Impactador de Partículas Dekati PM-10, Estación GPS Trimble, Cámara de Nubes.

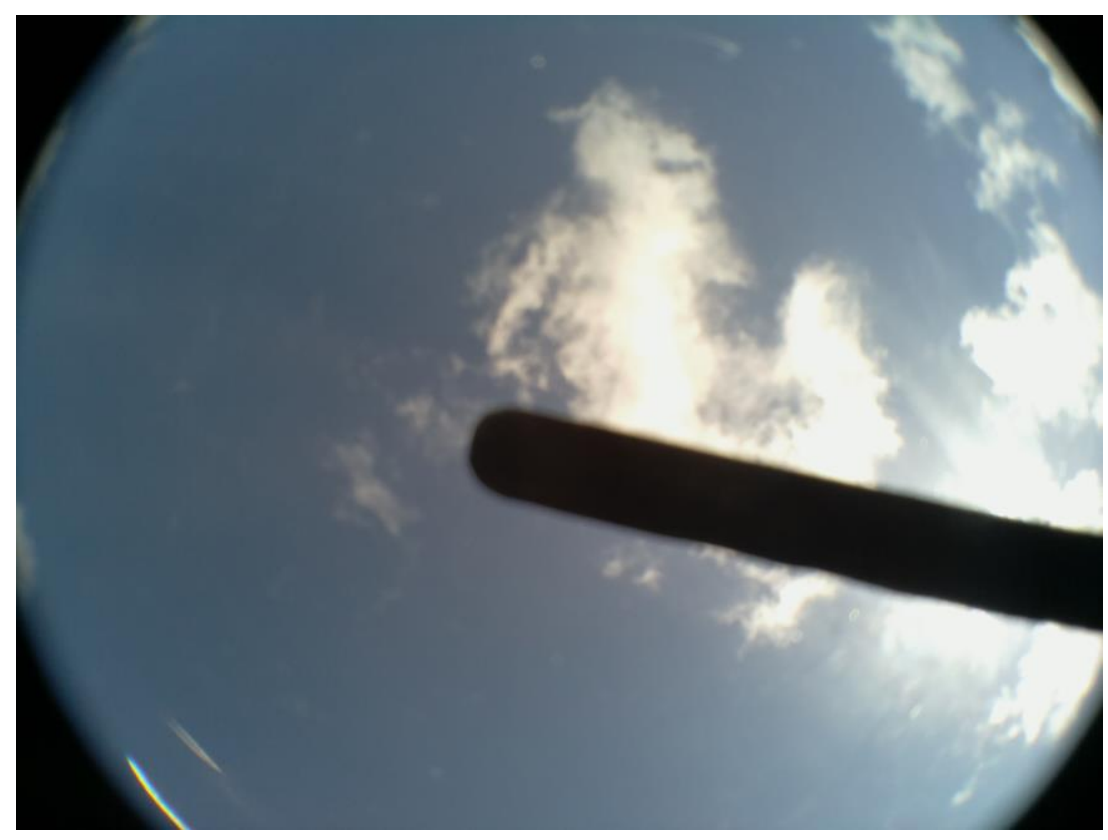

Fig.3. Imagen tomada con la cámara. Fecha 14 de agosto de 2015 a la hora 9:25.

\section{Conclusiones}

Se han presentado los resultados del diseño de un prototipo de cámara de nubes. Ha sido demostrada la posibilidad de construir este tipo de instrumento empleando una computadora miniaturizada consistente en una sola placa del tamaño de una tarjeta de crédito. La cámara de nubes diseñada cumple con los requisitos para un instrumento de este tipo. El instrumento en construcción debe convertirse en una alternativa de cámara de nubes barata para su uso extendido en países con escasos recursos. 


\section{Agradecimientos}

En esta primera experiencia de desarrollo de un prototipo de cámara de nubes ha sido fundamental la ayuda brindada por un grupo de colegas a los que nos referiremos a continuación. El MS. Albeth Rodríguez Vega y el Lic. Frank García Parrado, ambos pertenecientes al GOAC, por sus consejos y preocupaciones en el diseño de nuestro dispositivo. Juan Ricardo Lachicott, Yusniel Escalona Ramos y el Ing. Leonardo Fernández Jr., trabajadores del Centro Nacional de Radares por su aporte en recursos electrónicos y en el montaje de los mismos. Al Dr. John Braun del NCAR, USA, por proveernos el lente ojo de pez. Al Dr. Eliam Wolfram por sus valiosos consejos relacionados con el uso de lentes y cámaras. 